\title{
What we remember affects how we see: Spatial working memory steers saccade programming
}

\author{
Jason H. Wong • Matthew S. Peterson
}

Published online: 24 October 2012

(C) Psychonomic Society, Inc. 2012

\begin{abstract}
Relationships between visual attention, saccade programming, and visual working memory have been hypothesized for over a decade. Awh, Jonides, and ReuterLorenz (Journal of Experimental Psychology: Human Perception and Performance 24(3):780-90, 1998) and Awh et al. (Psychological Science 10(5):433-437, 1999) proposed that rehearsing a location in memory also leads to enhanced attentional processing at that location. In regard to eye movements, Belopolsky and Theeuwes (Attention, Perception \& Psychophysics 71(3):620-631, 2009) found that holding a location in working memory affects saccade programming, albeit negatively. In three experiments, we attempted to replicate the findings of Belopolsky and Theeuwes (Attention, Perception \& Psychophysics 71 (3):620-631, 2009) and determine whether the spatial memory effect can occur in other saccade-cuing paradigms, including endogenous central arrow cues and exogenous irrelevant singletons. In the first experiment, our results were the opposite of those in Belopolsky and Theeuwes (Attention, Perception \& Psychophysics 71(3):620-631, 2009), in that we found facilitation (shorter saccade latencies) instead of inhibition when the saccade target matched the region in spatial working memory. In Experiment 2, we sought to determine whether the spatial working memory effect would generalize to other endogenous cuing tasks, such as a central arrow that pointed to one of six possible
\end{abstract}

J. H. Wong $\cdot$ M. S. Peterson

Department of Psychology, George Mason University,

4400 University Drive MS3F5,

Fairfax, VA 22030-4444, USA

M. S. Peterson

e-mail: jason.h.wong@navy.mil

J. H. Wong $(\bowtie)$

Naval Undersea Warfare Center,

Building 1171/2, 1176 Howell Street,

Newport, RI 02841, USA

e-mail: jason.h.wong@navy.mil peripheral locations. As in Experiment 1, we found that saccade programming was facilitated when the cued location coincided with the saccade target. In Experiment 3, we explored how spatial memory interacts with other types of cues, such as a peripheral color singleton target or irrelevant onset. In both cases, the eyes were more likely to go to either singleton when it coincided with the location held in spatial working memory. On the basis of these results, we conclude that spatial working memory and saccade programming are likely to share common overlapping circuitry.

Keywords Attention and memory · Eye movements and visual attention

Visual attention, saccade programming, and visual working memory are all important areas of visual cognition that have been studied mostly in isolation, since each system has a different but related goal. Covert attention is the neural enhancement of perceptual processing due to tuning of the neural circuitry (Treue, 2001), whereas the enhanced processing from overt attention (eye movements) is due to shifting the eyes toward the object of interest. Overt attention also leads to enhanced neural processing, but this is a side effect of centering the object of interest on high-resolution foveal vision, as well as cortical magnification. Finally, visual working memory, unlike covert perceptual attention, is concerned with paying attention to internal, rather than external, representations (Cowan, 1995). There is strong evidence that these areas of the visual system overlap, however. By studying how the processes interact with each other, knowledge is uncovered about vision as a whole.

Some of the strongest evidence for a relationship between these systems is with covert visual attention and eye movements (Corbetta, 1998; Rizzolatti, Riggio, Dascola, \& Umiltà, 1987). Eye movements can be classified as voluntary or involuntary, with voluntary eye movements made in support of a task (e.g., saccades to a search target) and 
involuntary saccades made to an extraneous, task-irrelevant location. In support of theories proposing overlapping covert attention and saccade programming systems, research has shown that both voluntary and involuntary eye movements are preceded by a shift of attention to the saccade target. Additionally, when a saccade is cued to a particular location and then a target is displayed there as well, the target object is processed more quickly. This has been demonstrated for both voluntary (Deubel \& Schneider, 1996; Hoffman \& Subramaniam, 1995) and involuntary (Peterson, Kramer, \& Irwin, 2004) saccades, suggesting that the planning of an eye movement leads to the automatic shift of covert attention to the saccade destination prior to saccade execution.

Other research has demonstrated a relationship between visual object working memory and eye movements. Results show that remembering an object (e.g., a colored disk) can cause the eyes to make an involuntary saccade to that object if it appears in an unrelated search task (Olivers, Meijer, \& Theeuwes, 2006). Additionally, the rate at which memorized objects capture the eyes is equal to or stronger than an abrupt onset (Wong \& Peterson, 2011). Previous results demonstrating oculomotor capture involved salient environmental events, such as the abrupt onset of a new object or a change in object luminance. However, these results relating object memory and involuntary eye movements are unique, because oculomotor capture is occurring in part due to memory and not just bottom-up environmental factors.

In support of this is research suggesting that working memory and saccade preparation share circuitry. In a task comparing spatial working memory and saccade prospective memory, Srimal and Curtis (2007) found identical patterns of activation in the parietal cortex and frontal eye fields contralateral to the memorized location. This suggests that holding an item in working memory, even if there is no intention to make a saccade, activates areas that are involved in saccade planning. Similarly, Ikkai and Curtis (2010) found that neural activity in topographical areas in the prefrontal cortex and posterior parietal cortex was similar during spatial working memory, motor preparation, and spatial attention tasks (see also Curtis \& Connolly, 2008; Ikkai \& Curtis, 2008).

While efforts have been made to examine the effects of object memory on oculomotor capture, little is known about the effect of spatial memory on involuntary saccades. This relationship is the primary focus here. This research will also reexamine the effect of spatial memory on voluntary saccades. Results from previous studies differ from the results of research examining the effect of memorized locations on the visual system. Specifically, studies of spatial memory and attention suggest that remembering a location enhances attention at that location (Awh, Jonides, \& ReuterLorenz, 1998), while studies of spatial memory and voluntary saccades postulate an inhibitory effect on saccades toward the memorized location (Belopolsky \& Theeuwes, 2009).

A typical task examining the interaction between spatial memory and visual attention involves remembering the locations of several objects, followed by a delay period and then a recognition test to determine whether a location has moved. In an early study (Awh et al., 1998), probes were presented during the delay period between the memory display and memory test, and reaction time to detect those probes was shorter when they appeared at a memorized location. This result is consistent with attentional facilitation. Correspondingly, when the delay period consisted of a flickering checkerboard (Awh et al., 1999) or even nothing at all (Postle, Awh, Jonides, Smith, \& D'Esposito, 2004), fMRI and ERP results indicated that the visual cortex contralateral to the memorized locations was activated, indicating that attention-based rehearsal of the memorized locations leads to visual cortex activation. This corpus of results suggests that visual attention enables rehearsal in spatial working memory and creates attentional enhancement at that location.

Contrary to the effect of attentional enhancement at a memorized location, Theeuwes, Olivers, and Chizk (2005) demonstrated that saccades are inhibited when their paths intersect memorized locations. In this study, participants began each trial by remembering a location either above or below a central fixation cross. Then, when a signal was given, a saccade was made to a position above or below the cross so that the memorized location lay between the central cross and the saccade target. When the memorized location and saccade target were in the same vertical hemifield, the saccade path was curved around the memorized location, instead of passing directly through it.

Other recent research has shown that saccades made directly to a memorized location are initiated more slowly than saccades made away from the memorized location (Belopolsky \& Theeuwes, 2009). Participants were first asked to remember a location somewhere along the horizontal midline of the display. Afterward, a signal was given at the central fixation cross indicating whether to make a saccade to the side of the memorized location or the opposite side. When a saccade was made to the previously memorized location (or even to the same hemifield), participants were slower to execute that saccade.

The authors suggested that this inhibition is likely due to a mechanism known as inhibition of return (IOR), which biases attention away from previously visited locations (Klein, 2000). In the task, attention had recently visited the memorized location (in order to encode the location into memory) and was then moved to central fixation to wait for the cue, potentially leading IOR to inhibit a future saccade to the same location for a short period of time. This implies 
that attention first encodes the memorized location, IOR is automatically set up at that location, and then IOR affects saccades returning to the memorized location without affecting attention-based rehearsal. With this result, an increasing amount of evidence suggests that locations stored in working memory cause inhibition of saccades made near that location despite causing attentional facilitation.

The research here seeks to clarify the relationship between attention, eye movements, and working memory. Working memory is thought to utilize attention in location rehearsal, and other research has demonstrated the necessity of attention to plan a saccade. Logically, it follows that saccades should be primed to visit a memorized location because attention is already there. However, studies have shown that a memorized location inhibits saccades instead of attracting them. Examining how spatial memory can affect involuntary saccades (thought to be more automatic than the voluntary saccades that have been previously studied) and the rate of oculomotor capture can help clarify these conflicting results.

The following series of experiments examined how spatial memory affects oculomotor control. In all experiments, a location in space was presented for encoding; the participant then performed a saccade task and, at the end of the trial, was quizzed about the spatial location of the memorized item. The first experiment was an attempted replication of Belopolsky and Theeuwes (2009). Like Belopolsky and Theeuwes (2009), we found that the memorized spatial location affects saccade programming; however, we found facilitation in the form of shorter saccade latencies rather than inhibition. Experiment 2 used a central arrow to cue the location of the target. Unlike the first experiment, this asked participants to identify the saccade target with a button press. Again, we found that memory leads to faster saccades when the memorized location corresponds to the saccade target location. Finally, Experiment 3 was a visual search task in which the search target was a unique shape singleton (a square) among distractors (circles). To allow us to compare how spatial memory interacts with voluntary and involuntary saccade targeting, Experiment 3 also included a task-irrelevant abrupt onset. Again, we found facilitation when the memorized location corresponded to the target of the saccade, whether voluntary (square) or involuntary (onset).

\section{Experiment 1}

Method

\section{Participants}

A total of 7 George Mason University undergraduates received partial class credit in exchange for their participation
( 2 males, 5 females). The mean age of participants was 20.1 years, and all participants had normal or corrected-tonormal color vision.

\section{Apparatus}

A Mac Pro equipped with a 21-in. (20-in. viewable) monitor operating at $85 \mathrm{~Hz}$ with a resolution of $1,024 \times 768$ was used to display stimuli. This computer was networked to a Dell Pentium 4 PC that collected eye-tracking data in conjunction with an Eyelink 2 eyetracker (SR Research, Ontario, Canada). The Eyelink 2 system samples at a rate of $250 \mathrm{~Hz}$ and has a $0.2^{\circ}$ spatial resolution. The head was stabilized by means of a chinrest located $70 \mathrm{~cm}$ from the monitor.

\section{Stimuli}

The stimuli were identical to those used by Belopolsky and Theeuwes (2009), except for the mapping of the color spatial cue. In their study, the color red signified a saccade to the left, whereas the color green signified a saccade to the right. We switched the colors to red for right and green for left, since pilot testing found this mnemonic easier to learn.

\section{Design and procedure}

The design of this experiment was identical to that in Experiment 2 of Belopolsky and Theeuwes (2009). Each trial began with an encoding screen that lasted for $2,500 \mathrm{~ms}$. Two circles $1.35^{\circ}$ in diameter appeared $4^{\circ}$, $4.8^{\circ}$, or $5.6^{\circ}$ to the left and to the right of a central fixation dot. During the first $1,000 \mathrm{~ms}$ of the encoding screen, a line at fixation pointed to the left or right, indicating which circle location to memorize. This was followed by either a 1,000- or a 2,000-ms blank interval in which only a central fixation dot was present. Following the blank interval, a cue screen occurred for 2,000 ms. On the cue screen, crosses appeared to the left and right of fixation at the location where the circles had appeared in the encoding screen. The central dot changed color to green, indicating that the eyes should saccade to the left cross, or red, indicating that the eyes should saccade to the right cross. The cue screen was followed by a $1,000-$ or $2,000-\mathrm{ms}$ blank screen in which the eyes had to move back to fixation. Finally, a memory probe screen appeared in which a single location to the left or right was circled. On half the trials, the probe coincided with the target; on the remaining trials, the probe was $0.7^{\circ}, 1.2^{\circ}$, or $1.7^{\circ}$ to the left or right of the original memory location. The task of the participant was to maintain central fixation and respond with a button press whether the probe coincided with the memorized location. 
Participants completed three blocks of 48 trials, for a total of 144 trials. The side of the memorized object as well as the distance of the memorized object from fixation $\left(34^{\circ}, 4.8^{\circ}\right.$, or $5.6^{\circ}$ ) occurred equally often and were completely crossed. The memory probe always occurred on the encoding side but coincided with the memorized location at chance level. On mismatch trials, the probe occurred in one of the other two possible locations on the side that was encoded. On half the trials, the saccade was directed to the same side that the memory probe occurred (congruent trials); on the remaining trials, the eyes were directed in the opposite direction (incongruent trials). Finally, the two encoding delays occurred equally often (1,000 or 2,000 ms).

\section{Results}

An average of $5.2 \%$ of the trials were discarded because participants did not remain fixated at the center of the screen before the onset of the saccade cue display.

\section{Memory and saccade task performance}

Across experimental conditions, $t$-tests were performed for memory accuracy, saccade accuracy, and saccade reaction times (SRTs). For the SRTs, latencies less than $100 \mathrm{~ms}$ and longer than 1,200 ms were initially discarded. After this initial filtering, any SRTs more than two standard deviations from each condition's mean were discarded for each participant. In addition, only SRTs from trials on which participants correctly responded to the memory probe were analyzed.

Saccade targeting was more accurate when the saccade target was on the same side as the memorized location, as compared with when it was on the opposite side $(M=79 \%$ and $69 \%), t(6)=2.5, p<.05$. Accurate saccades had a shorter latency when they were made to the memorized side (congruent), as compared with when they had to be made to the opposite side $(M=451$ and $468 \mathrm{~ms})$, one-tailed $t(6)=$ $2.0, p<.05$. Memory accuracy was not affected by saccadememory congruency $(M=69 \%), t(6)=1.3, p>.1$.

\section{Discussion}

Like previous findings, we found that holding a spatial location in working memory affected saccade programming-namely, saccade accuracy and saccade latency. However, unlike in Belopolsky and Theeuwes (2009), our effect was in the opposite direction: Rather than memory inhibiting saccade programming, we instead found that memory facilitates saccade programming. Our results are in line with a number of findings, such as those of Awh and colleagues (1998), who found that spatial working memory was impaired when attention was shifted to another location, suggesting that spatial shifts of attention mediate spatial working memory. Similarly, Everling and colleagues found that macaques were more likely to make erroneous prosaccades in an antisaccade task when the prosaccade location showed increased prestimulus activity (Everling, Dorris, \& Munoz, 1998; Everling \& Munoz, 2000). On average, our saccade latencies were approximately $150 \mathrm{~ms}$ shorter than the average reported by Belopolsky and Theeuwes (2009); perhaps the longer delay allows more time for IOR to develop.

\section{Experiment 2}

The goal of Experiment 2 was to determine whether we could find spatial working memory affecting saccade targeting in other tasks. In this case, we were interested in examining central arrow cuing tasks - specifically, those that use multiple locations and require the participant to identify a target at one of those locations. We chose this type of task not only because it had a long history (Cheal \& Lyon, 1991a, b; Müller \& Rabbit, 1989; Yantis \& Jonides, 1990), but also because it allowed us to examine the effects of multiple competing stimuli and allowed us to have a condition in which the memorized location coincided exactly with the location of the saccade target.

\section{Method}

\section{Participants}

A total of 16 George Mason University undergraduates received partial class credit in exchange for their participation (4 males, 12 females). The mean age of participants was 20.7 years, and all participants had normal or corrected-tonormal color vision.

\section{Apparatus}

The apparatus was identical to that used in Experiment 1.

Stimuli

Figure 1 shows a stylized version of the stimuli used in Experiment 2. The location to be memorized was indicated by a black dot that was $0.95^{\circ}$ in diameter. This dot was used during the memory display and also appeared in the memory test display. In the no-memory condition, the memory display had the message "no memory" displayed in the center. For the memory test display, three dots were presented, each $2.86^{\circ}$ apart. For the search task, target and distractors were gray circles measuring $3.90^{\circ}$ in diameter, with the target indicated by a central arrow $2^{\circ}$ in length. Each search 
display consisted of six objects: five distractors and one target. The shapes were located $9.33^{\circ}$ away from the center of the screen, arranged around an invisible circle, with $60^{\circ}$ of angular distance between each object. Within each search distractor, an up- or down-facing $\mathrm{C}$ was present. Within the search target, a left- or right-facing $\mathrm{C}$ was present. These figures were colored black, centered within each object, and $0.03^{\circ} \times 0.03^{\circ}$ in size. The figures inside each search object were too small to be discriminated by peripheral vision and required direct fixation.

A search preview screen occurred immediately before the search display. This display consisted of six square-circle hybrids located in the same position as the stimuli in the search display. This display provided a placeholder for the search objects. In this experiment, the search preview and search display always contained six objects arranged in a circle, but these six objects could be located in one of two configurations (differing by the physical location of objects in the display).

The difference between these configurations was a $30^{\circ}$ radial offset between all search objects in the two configurations. One of these two configurations was chosen at random for each trial. This was necessary due to methodological concerns. Since the memorized location could coincide with a search object or an abrupt onset, consistently located search objects would lead to the memorized location indicating the eventuality of an abrupt onset at its location. This is because it could be simple to deduce when the memorized location was not at a regular search object location but at an onset location instead. Varying the locations of the search objects from trial to trial prevented this from occurring.

\section{Design and procedure}

This experiment contained one within-participants manipulation: the relationship between the memorized location and the search objects. This led to four conditions: away from the search array (along the edges of the display), at the search target, at a search distractor, and no memory. The memorized location corresponded to a stimulus location $75 \%$ of the time, and of that $75 \%$, it corresponded to the target at chance level (1/6). Half of the remaining $25 \%$ of the trials consisted of either no-memory trials or trials in which the memory item was on a nonstimulus location. There were a total of 288 trials in the experiment. The entire experiment took approximately 1 hour to complete.

Each trial consisted of five main displays (see Fig. 1). The first display consisted of a small black disk presented for 1,000 ms, which participants were asked to remember. The location of the memorized disk corresponded to the appropriate condition (away, target, distractor). In the case of the no-memory condition (not shown in Fig. 1), no disk appeared, and a message occurred at fixation stating "no memory." Participants were asked to keep their eyes at the center of the screen. The second display was a $750-\mathrm{ms}$ search preview screen where the eyes were to remain fixated on the center cross. If the eyes deviated from the center cross, an error message was displayed, and the trial was aborted. This screen contained six hybrid square-circle objects as placeholder masks for the eventual search objects. This display, as well as the search display, was organized in one of two configurations so as to minimize the probability of participants being positionally primed to possible onset locations.

Participants then saw the search display containing the target and five distractors. In the search display, the fixation cross was replaced by an arrow cue indicating the location of the target. Participants were tasked with making a saccade to the target as quickly as possible while making as few eye movements as possible. Within the target circle was a left- or right-facing $\mathrm{C}$, and participants responded as to which figure was present with a buttonpress. The remaining distractor circles contained Cs open at the top or bottom. After the participant manually responded, the search display was replaced with a fixation cross for $1,000 \mathrm{~ms}$. Finally, the memory test display placed three black dots next to each other $2.86^{\circ}$ apart, and participants responded as to which dot was in the memorized location while fixating the center of the screen.

\section{Results}

An average of $6 \%$ of the trials were discarded because participants did not remain fixated at the center of the screen before the onset of the search display. For the SRTs, latencies less than $100 \mathrm{~ms}$ and longer than 1,200 ms were initially discarded. For each participant, all saccade latencies that were more than two standard deviations away from each cell's mean were discarded.

\section{Search task performance}

A one-way repeated measures ANOVA with four levels was performed across experimental conditions for manual search accuracy and response times. The type of memory task did affect manual accuracy, $F(3,48)=4.06, p<.05$, and a post hoc contrast demonstrated that this was driven by the memorized distractor condition, $F(1,16)=15.0, p<.01$ ( $M=82 \%$ and $87 \%$ for the memorized distractor and remaining conditions, respectfully). The four different memory conditions had no effect on manual response times to the search target, $F(3,48)<1, M=921 \mathrm{~ms}$.

To examine whether the location of the memory item affected eye movements, we examined both latency of 




Fig. 1 Trial schematic for Experiment 2, consisting of both the memory and search tasks. Here, the memorized location is that in the away condition and does not correspond to the target or distractor locations.

saccades and accuracy of fixations to the target for trials on which the memory task was performed correctly (see Fig. 2). For the latency data, we were primarily interested in whether latencies to the target differed depending on whether the memorized item was at the target, as compared with the other locations. A fixation was determined to be on an object if (1) the eyes were not making a saccade and (2) the eyes were no more than $4.68^{\circ}$ from the center of the object (which was $3.90^{\circ}$ in diameter), and (3) consecutive fixations on the same object were considered a single fixation. Eye movements were classified as saccades if (1) they exceeded a speed greater than $30 \mathrm{deg} / \mathrm{s}$ and acceleration exceeded $8000 \mathrm{deg} / \mathrm{s}^{2}$ or (2) acceleration exceeded $8,000 \mathrm{deg} / \mathrm{sec}^{2}$ and the distance exceeded $0.2^{\circ}$.

A repeated measures ANOVA contrast found that saccades to the target were significantly quicker when the memorized location occurred at the target location, as compared with the other conditions, $F(1,16)=6.28$, $p<.05$ ( $M \mathrm{~s}=377$ and $398 \mathrm{~ms})$. Subsequent one-tailed $t$-tests revealed that when the memorized item was at the target, latencies to the target were significantly quicker than when the memorized item was at a distractor, $t(16)=2.8, p<.017$, or away from the main display, $t(16)=2.4, p<.017$.

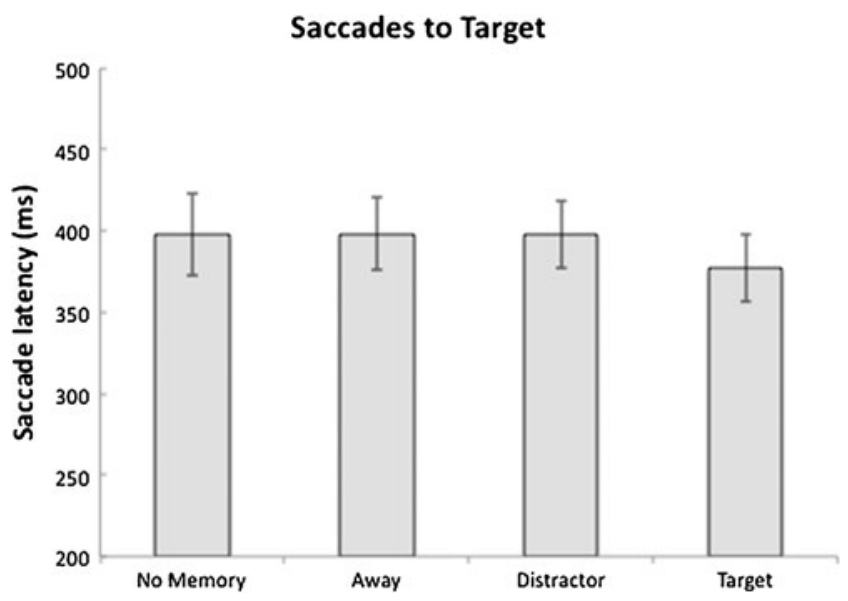

Fig. 2 Saccade latencies (SRTs) for Experiment 2
In the no-memory condition, the screen displayed the message "no memory." The cued saccade location is at 2 o'clock. One stimulus configuration is shown. Displays and stimuli are not to scale

Shown in Fig. 3 are the fixation accuracy data-the percentage of initial fixations that landed on the target. A one-way repeated measures ANOVA analysis of the search accuracy data found that the type of condition affected fixation accuracy, $F(3,48)=4.13, p<.05$. A post hoc contrast of the distractor condition versus the remaining conditions found that saccades were less likely to go to the target when the memorized location was at a distractor, $F(1$, $16)=12.4, p<.01$. In order to test whether this was due to increased capture by memorized distractors, a contrast was performed comparing trials on which the memorized location was a distractor with those in the remaining three conditions. Distractor fixation rates were higher when the distractor was the memorized location, $F(1,16)=6.31$, $p<.05(M=5.4 \%)$, as compared with the remaining three conditions $(M=3.6 \%)$.

\section{Saccadic curvature for eye movements to targets}

Saccade curvature was examined by finding the maximum angular deviation from a straight line from central fixation to the target object. A one-way ANOVA with four levels found no significant difference between the four memory



Fig. 3 Percentage of initial fixations landing on the target or on a distractor for Experiment 2. Error bars are included only for fixations on the target 
conditions $\left(M=12.01^{\circ}\right), F(1,16)=0.82$. This indicates that the location of the memorized item had no effect on saccade curvature.

\section{Memory task performance}

Figure 4 shows accuracy in the memory task contingent on whether the memory item was away from the search objects, on a distractor, or on a target. A oneway repeated measures ANOVA performed on the data for the three conditions that required memorization revealed a main effect of condition, $F(2,32)=14.7$, $p<.01$. Subsequent one-tailed $t$-tests revealed that memory accuracy was significantly lower in the away condition than in the distractor condition, $t(16)=2.8$, $p<.01$, and the distractor condition produced significantly lower accuracy than did the target condition, $t$ $(16)=4.7, p<.01$.

\section{Discussion}

As in Experiment 1, we found that holding an item in working memory attracted saccades to that location. In the case of Experiment 2, this led to shorter saccade latencies to the target when the memorized location coincided with the target. Likewise, the memorized location could have a distracting effect: When it coincided with a distractor, it led to fewer saccades to the target and more to the distractor that coincided with the memorized location. Taken as a whole, this suggests that holding a location in working memory can prime saccades and, depending on the goal of the saccade, can lead either to faster saccades to the saccade targets or to capture by irrelevant distractors.

\section{Memory when the eyes went to the target}

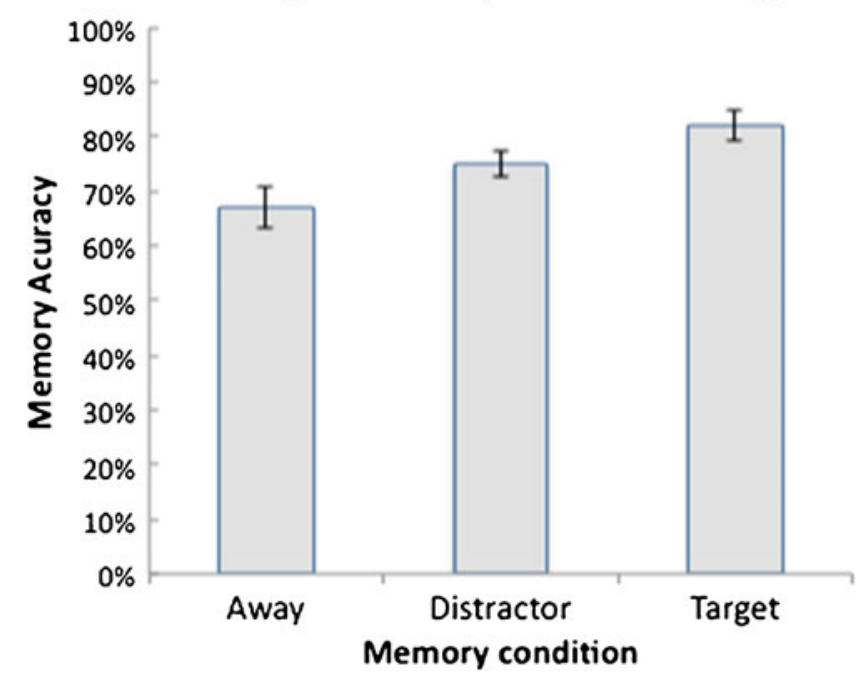

Fig. 4 Memory accuracy for Experiment 2

\section{Experiment 3}

The goal of Experiment 3 was twofold. The first goal was to determine whether spatial memory can affect involuntary attentional capture-in this case, by abrupt onsets. The second goal was to determine whether spatial memory can affect visual search. This is a critical difference between Experiment 3 and the first two experiments. In those experiments, the saccade target was cued by a cue at fixation, whereas in Experiment 3, the response target was located in a shape singleton. This required participants to spread their attention peripherally in order to detect the location of the target and plan their saccade.

Method

\section{Participants}

A total of 15 George Mason University undergraduates received partial class credit in exchange for their participation ( 3 males, 12 females). The mean age of participants was 19.9 years, and all participants had normal or corrected-tonormal color vision.

\section{Apparatus}

The same apparatus as that used in Experiments 1 and 2 was used for Experiment 3.

\section{Stimuli}

Other than the following differences, the stimuli were identical to those used in Experiment 2. For the search task, distractors were gray circles measuring $3.90^{\circ}$ in diameter, and the target was a gray square of the same size. Each search display consisted of six objects: five distractors and one target. With each search display, a new object could abruptly onset between two other search objects. This new object was a gray circle and was identical to a search distractor.

A search preview screen occurred immediately before the search display and was identical to that used in Experiment 2. This display provided a placeholder for the search objects and allowed for the abrupt onset of a new object where there was no previous placeholder.

\section{Design and procedure}

This experiment contained two within-participants manipulations: the relationship between the memorized location and the search objects (away from the search array, at the search target, at a search distractor, or at the abrupt onset) and whether an abrupt onset was present or absent. These 
two factors were crossed to create the experimental conditions. The control conditions in this experiment occurred when the memorized location either coincided with a search distractor or was away from the search array (i.e., in one of the corners of the screen). It must be noted that the memorized location could not coincide with an abrupt onset if the onset was absent from the trial. Therefore, this one condition was not possible, leaving a total of seven conditions (i.e., there was no onset-absent/location-at-onset condition).

There was a total of 300 trials in the experiment. The entire experiment took approximately 1 hour to complete. Table 1 lists how many trials of each condition were contained in this experiment. It is important to note that the ratios of trial conditions are necessary to ensure that the memorized location does not prime likely locations of the target or onset. This is especially true of the 5:1 ratio between trials with the memorized location at the distractor and trials with memorized location at the target. This proportion was used because there were five distractors in the search display, as compared with only one target.

Each trial consisted of five main displays (see Fig. 5). The first display consisted of a small black disk presented for $1,000 \mathrm{~ms}$, which participants were asked to remember. The location of the memorized disk corresponded to the appropriate condition (away, target, distractor, or onset). Participants were asked to keep their eyes at the center of the screen. Following this display was a 2,000-ms search preview screen where the eyes were to remain fixated on the center cross. This screen contained six hybrid square-circle objects as placeholder masks for the eventual search objects; this preview was needed so that a new object could be abruptly onset (if included in the search display) where there was no object before.

This display was organized in one of two configurations so as to minimize the probability of participants being positionally primed to possible onset locations.

Participants then saw the search display containing a square target and five gray circle distractors. If an abrupt onset was present, a new object identical to a circular search distractor was presented in between two search objects. Target and onset locations were randomized across all trials. Participants were tasked with making a saccade to the square target as quickly as possible while making as few eye movements as possible. Within the square was an open

Table 1 Experimental conditions generated by crossing the variables of onset presence and location coincidence (cells represent number of trials for each condition)

\begin{tabular}{lllll}
\hline & $\begin{array}{l}\text { Location away } \\
\text { from objects }\end{array}$ & $\begin{array}{l}\text { Location } \\
\text { at distractor }\end{array}$ & $\begin{array}{l}\text { Location } \\
\text { at target }\end{array}$ & $\begin{array}{l}\text { Location } \\
\text { at onset }\end{array}$ \\
\hline Onset present & 30 trials & 75 trials & 15 trials & 60 trials \\
Onset absent & 30 trials & 75 trials & 15 trials & N/A \\
\hline
\end{tabular}

box figure, and participants responded as to which figure was present. Finally, the memory test display placed three black dots next to each other $2.86^{\circ}$ apart, and participants responded as to which dot was in the memorized location.

\section{Results}

An average of $5.75 \%$ of trials were discarded because participants did not remain fixated at the center of the screen before the onset of the search display.

\section{Memory and search task performance}

A one-way ANOVA with seven levels was performed across experimental conditions for memory accuracy, search accuracy, and search reaction times (see Table 2 for means for each condition). The memory accuracy analysis showed an effect of condition, $F(6,84)=6.37, p<.001$. Contrasts suggested that memory accuracy did not differ depending on the presence or absence of an onset $(M=71.63 \%, S E=4.10$, for onset absent; $M=71.85 \%, S E=3.25$, for onset present), $F$ $(1,14)=0.13, p=.91$. However, memory accuracy increased when the memorized location was at the target (regardless of onset presence), as compared with when the location was elsewhere (all other conditions; $M=79.65 \%, S E=4.70$, for target at location; $M=68.60 \%, S E=3.18$, for other conditions), $F(1,14)=15.19, p<.001$. This result suggests that visiting the target when it coincided with the memorized location increased memory accuracy.

Memory accuracy did not increase when the memorized location was at the onset. Performance in the memory task was no different when the memorized location was at the onset versus when it was at a distractor or away from the search objects $(M=68.80 \%, S E=2.50$, for onset at location; $M=68.55 \%, S E=3.35$, for other conditions), $F$ $(1,14)=0.28, p=.60$. To further investigate whether saccade destination interacted with memory, we analyzed memory performance when the eyes went to a nontarget location on the basis of whether that location matched the memory location or not. None of the main effects (memory location and saccade/memory match/mismatch) were significant (largest $F=0.769$ ), nor was the interaction between memory location and saccade destination, $F(2,24)=2, p>.15$. Therefore, fixating the target when it coincided with the memorized location improved memory accuracy, but fixating other memorized locations did not.

Search accuracy did not differ across conditions, $F(6,84)=$ $0.30, p=.93$, although search reaction times did differ, $F(6$, $84)=3.83, p<.01$. Reaction times were not affected by the presence of an abrupt onset $(M=1,015.87 \mathrm{~ms}, S E=79.93$, for onset absent; $M=1,042.49 \mathrm{~ms}, S E=68.13$, for onset present), $F(1,14)=1.25 p=.27$, but search was faster when the target 


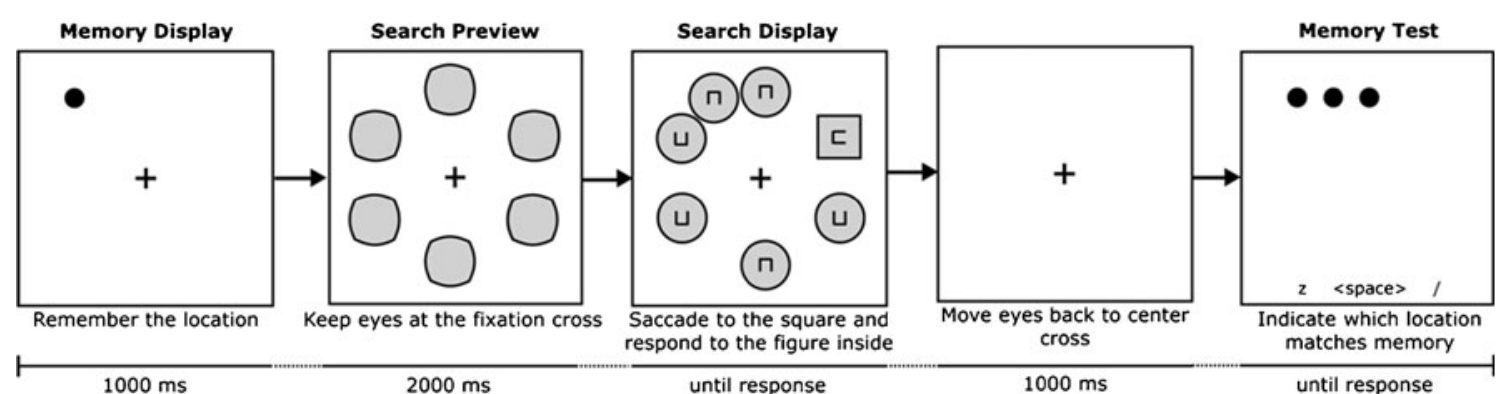

Fig. 5 Trial schematic for Experiment 3, consisting of both the memory and search tasks. Here, the memorized location coincides with the abrupt onset. One stimulus configuration is shown. Displays and stimuli are not to scale

was at the memorized location $(M=950.70 \mathrm{~ms}, S E=78.88$, for target at location; $M=1,063.24 \mathrm{~ms}, S E=70.91$, for other conditions), $F(1,14)=20.60, p<.001$. This result suggests that the memorized location was able to attract to the eyes to the target and make responses to the search task faster.

\section{First fixations to search objects}

Fixations were examined when the memorized location coincided with the target or onset to determine whether fixations to the search stimulus were increased beyond a no-memory baseline. In order to examine cooperation between the target and memorized location, a $2 \times 3$ ANOVA was conducted with onset presence and memorized location relationship (whether the location was away from the search display or coincided with the distractor or target) as factors (Fig. 6). The dependent variable in this analysis was percentage of first fixations to the target, as compared with the total number of fixations made to other search objects. There was an effect of onset presence, $F(1$, $14)=24.12, p<.001$, and a trend toward an effect of memorized location relation, $F(2,28)=3.13, p=.06$. There was no interaction between the two factors, $F(2,28)=0.89, p=.42$.

Contrasts (collapsed across onset presence) were performed to further examine the effect of fixations to the search target when it coincided with the memorized location. The eyes were significantly more likely to first saccade to the target at the memorized location than to the target when the location was elsewhere $(M=82.10 \%, S E=3.80$, for fixations to target at location; $M=75.58 \%, S E=4.08$, for fixations to target when the location was elsewhere), $F$ $(1,14)=18.79, p<.001$.
A similar analysis was done for first fixations on the onset. An ANOVA performed on the four different memorized location conditions (away, distractor, target, onset) when the onset was present (Fig. 6). There was a significant effect of condition, $F(3,42)=3.87, p<.05$, and contrasts between first-fixation rates when the location was at the onset and when the location was elsewhere suggest that the eyes were more likely to first visit the onset when it was at the memorized location $(M=20.70 \%, S E=3.20$, for fixations to location at onset; $M=12.77 \%, S E=2.50$, for fixations to onset when the location was elsewhere), $F(1$, $14)=10.16, p<.01$. Therefore, both the onset and the target received more first fixations when they shared their position with the memorized location.

It is also important to examine the frequency with which the memorized location was able to draw the eyes to a nonunique distractor (see Fig. 7) or to the away location. Distractors do not often receive the first fixation, since they are not salient to the visual system nor are they task relevant. One possibility is that the memory effect observed for the onsets and targets is simply an additive effect; that is, the effect might not be due to the memory priming the location but, rather, could be due to the memory having an independent effect. If that is the case, we should find that the percentages of saccades that go to the memorized distractor and away locations should increase by similar rates. The fact that no saccades were made to the away location would argue against this, but a case could be made that saccades were not made because the away location did not contain an object. To test whether the memory effect was additive or whether it interacted with the type of object, separate

Table 2 Behavioral data (and standard errors) for all conditions

\begin{tabular}{|c|c|c|c|c|c|c|c|}
\hline & Away/ Absent & Away/ Present & Dist/ Absent & Dist/ Present & Target/Absent & Target/ Present & Onset/ Present \\
\hline Memory accuracy & $63.89(2.93)$ & $69.33(3.27)$ & $69.68(3.69)$ & $71.29(3.45)$ & $81.35(5.75)$ & $77.98(3.70)$ & $68.78(2.53)$ \\
\hline Search accuracy & $91.22(1.38)$ & $90.02(1.24)$ & $91.23(1.11)$ & $91.13(1.34)$ & $90.73(1.31)$ & $91.93(2.04)$ & $91.40(1.13)$ \\
\hline Search RT & $1,045.63(63.19)$ & $1,024.25(71.58)$ & $1,073.26(86.79)$ & $1,111.22(71.31)$ & $950.11(81.41)$ & $951.27(76.34)$ & $1,061.83(61.68)$ \\
\hline
\end{tabular}

Note. Each column is a different experimental condition, defined by memorized location relation (away, distractor [Dist], target, onset) and onset presence or absence. Memory and search accuracy are reported as percent correct, and search reaction time is reported in milliseconds. 
Fig. 6 First fixations to the target (top half) or onset (bottom half), grouped by memorized location condition. The target fixation data are also grouped by onset presence or absence. The memorized location combined with either the target or onset led to more first fixations to that search object

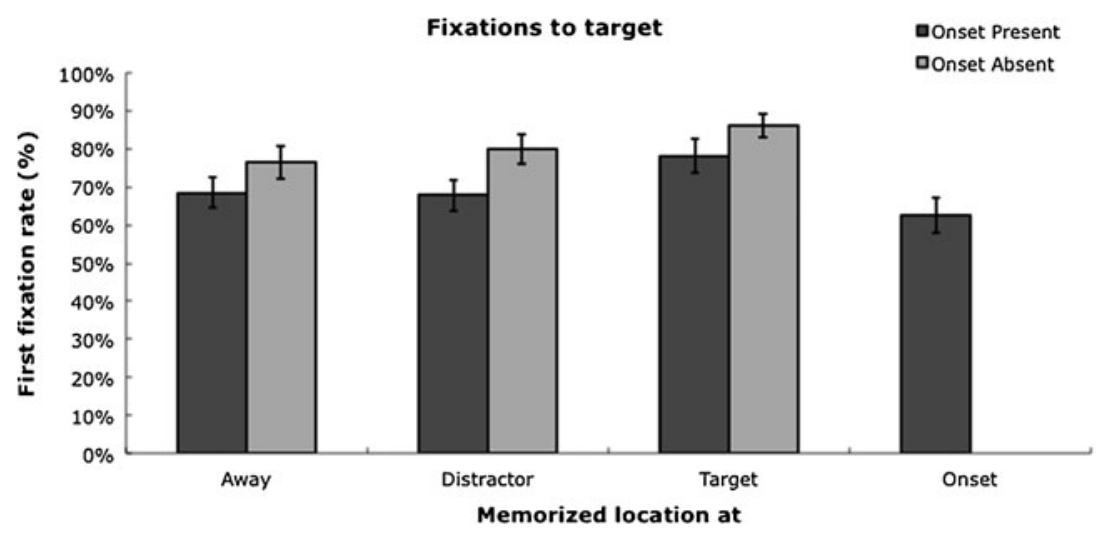

Fixations to onset

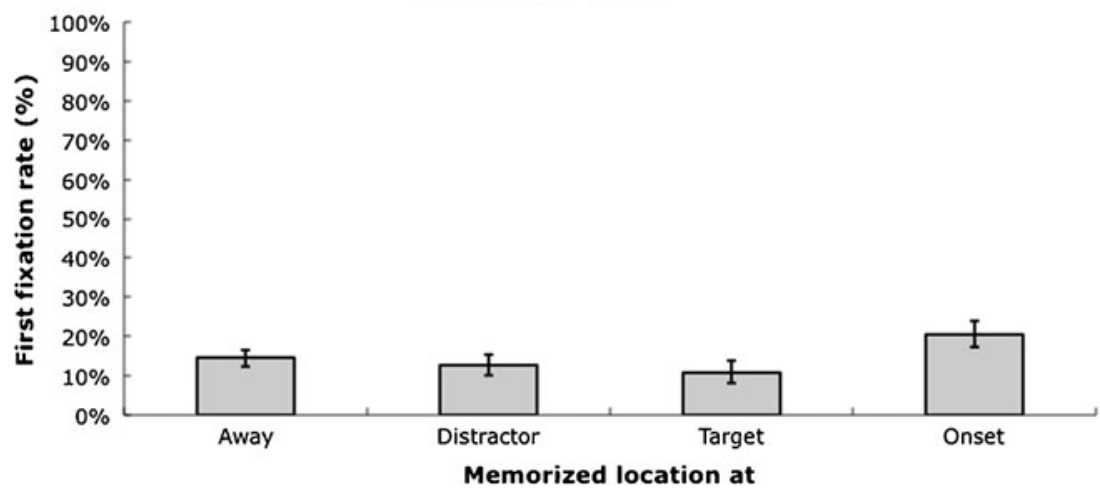

ANOVAs (excluding the away condition) were run for when the onset was absent $(2 \times 2$; distractor and target locations $)$ and when the onset was present $(2 \times 3$; distractor, target, and onset locations). When the onset was absent (left panel of Fig. 7), there was a main effect of whether the location was memorized, $F(1,14)=113.5$, and a main effect of the type of item at the location, $F(1,14)=93.4$, and the two interacted, $F(1,14)=240.0$, all $p \mathrm{~s}<.0005$. Likewise, when the onset was present (right panel of Fig. 7), there was a main effect of whether the location was memorized, $F(1,14)=$ 13.7 , and a main effect of the type of item at the location, $F$ $(2,28)=300.7$, and the two interacted, $F(2,28)=87.0$, all $p s<.01$. These interactions indicate that the onset effect is not simply additive in nature.

\section{Influence of memorized location on voluntary} versus involuntary saccades

Competition between the target and abrupt onset for the first fixation when one object coincided with the memorized location was examined. This provided evidence to determine whether a combined stimulus (e.g., memorized location at onset) "stole" fixations away from the other object (e.g., search target). A $t$-test was conducted where fixations
Fig. 7 Mean percentage of first fixations to various regions of interest depending on whether the region of interest contained the memorized item (light bar) or not (dark bar). The right panel shows the data for when an onset is present in the display, and the left panel is for onset-absent trials. For both sets of data, significant interactions were found between region of interest and whether the region was memorized or not
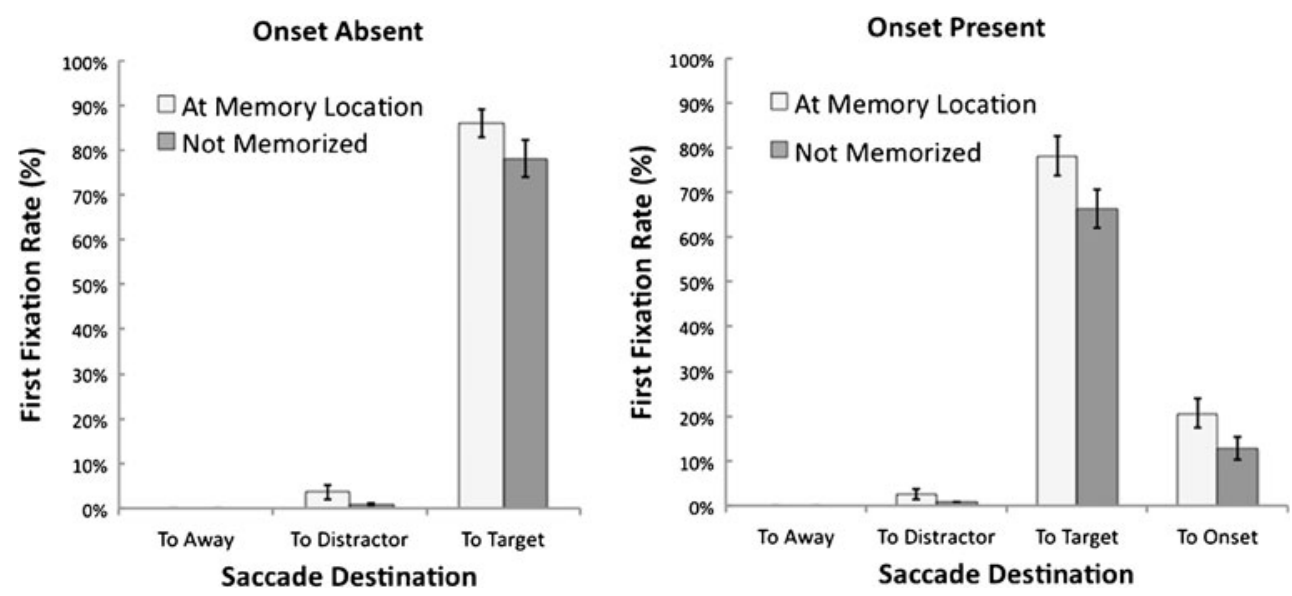
to the target were examined between conditions where the memorized location coincided with the onset or a distractor. The latter condition served as a control because the distractor at the memorized location should not affect fixations to the onset or target. The analysis confirmed that the coincidence of the onset and memorized location led to fewer fixations to the target, as compared with a control condition $(M=67.77 \%, S E=4.15$, for target fixations when the distractor coincided with the location; $M=62.52 \%, S E=$ 4.60 , for target fixations when the onset coincided with the location), $t(14)=2.28, p<.05$. Therefore, the coincidence of the onset at the memorized location led to fewer initial saccades to the target.

A similar $t$-test was conducted to determine whether the onset received fewer fixations when the memorized location coincided with the target. This did not occur $(M=12.71 \%, S E=$ 2.55 for onset fixations when the distractor coincided with the location; $M=10.94 \%, S E=2.77$, for target fixations when the onset coincided with the location), $t(14)=0.49, p=.63$. The onset and memorized location combination drew fixations away from the target. However, the target and memorized location combination did not draw fixations away from the onset. This result suggests that spatial memory affects saccade programming differently for voluntary and involuntary saccades, possibly due to the temporal differences in processing onsets and targets.

\section{Saccadic latencies for eye movements to search objects}

The influence on saccadic latencies was examined for possible excitatory or inhibitory effects on the visual system. A $2 \times 3$ ANOVA of the effects of onset presence and memorized location relation (away, distractor, target) on the latencies of saccades to the target was conducted (Fig. 8a). While onset presence had no effect, $F(1,14)=0.28, p=.60$, memorized location relation did, $F(2,28)=8.95, p<.001$. There was no interaction between these two factors, $F(2,28)=$ $1.30, p=.29$. Linear contrasts demonstrated that saccades were initiated to the target faster when it coincided with the memorized location than when it did not $(M=381.48 \mathrm{~ms}$, $S E=40.71$, for fixations to the target at location; $M=$ $433.78 \mathrm{~ms}, S E=20.81$, for target fixations when the location was elsewhere), $F(1,14)=17.14, p<.001$, suggesting that the memorized location aided the target in drawing the eyes more quickly.

Saccadic latencies to the onset were examined using an ANOVA for the four memorized location conditions that contained an onset during search (Fig. 8b). There was no effect of condition, $F(3,27)=1.72, p=.19$, suggesting that the eyes did not saccade to the onset sooner when the onset was present at the memorized location. Nonetheless, across all conditions, saccades to the onset were made sooner than saccades to the target regardless of where the memorized location was $(M=416.35 \mathrm{~ms}, S E=27.44$, for saccades to the target; $M=305.00 \mathrm{~ms}, S E=18.90$, for saccades to the onset), $t(14)=12.82, p<.001$. This indicates that abrupt onsets are able to cause the fastest saccade initiation.

Finally, latencies were used to determine whether the memorized location at the target or onset slowed saccades to the other relevant search object. $T$-tests showed that saccades were made no more slowly to the target when the memorized location coincided with the onset versus the control condition of when the location coincided with a search distractor, $(M=440.41 \mathrm{~ms}, S E=19.48$, for onsets at the memorized location; $M=460.02 \mathrm{~ms}, S E=25.87$, for distractors at the memorized location), $t(14)=1.66, p=.12$. This pattern also held for saccades to the onset when the memorized location coincided with the target versus when it coincided with a distractor $(M=300.32 \mathrm{~ms}, S E=14.67$, for targets at the memorized location; $M=326.82 \mathrm{~ms}, S E=$ 30.73 , for distractors at the memorized location), $t(14)=$ $1.02, p=.34$. Therefore, when the memorized location coincided with the abrupt onset or target, saccades were not slower, as compared with when they were made to the other relevant object.

\section{Saccadic curvature for eye movements to targets}

Saccade curvature was examined by finding the maximum angular deviation from a straight line from central fixation to the target object. A one-way ANOVA with seven levels (onset present or absent when the memory item was a distractor, target, or away, as well as when the memorized item was the onset) found no significant difference between the seven conditions $\left(M=14.63^{\circ}\right), F(1,6)=0.51$. This indicates that the location of the memorized item had no effect on saccade curvature.

\section{Discussion}

This experiment examined the relationship between a memorized location, a search target, and the abrupt onset of a new object. The interaction of these three stimuli allowed spatial memory to interact with voluntary eye movement planning (for saccades to the search target) and involuntary planning (for saccades to the abrupt onset). As in Experiments 1 and 2, we found that memorizing a spatial location tended to prime that area, making saccades to the memorized location more likely to occur. Additionally, saccades made to the target were executed more quickly when the target coincided with the memorized location than when it did not. These are not simply additive effects; that is, the effect was not due to additional memory-guided saccades, since saccades were rarely, if ever, made to the memorized distractor or "away" locations (see Fig. 7). Instead, these results suggest that the spatial memory is able to prime the 
Fig. 8 Saccadic latencies to the target (top half) or onset (bottom half), grouped by memorized location condition. The target fixation data are also grouped by onset presence or absence. Latencies to the target decreased when the target was at the memorized location, but no such effect was found for the onset
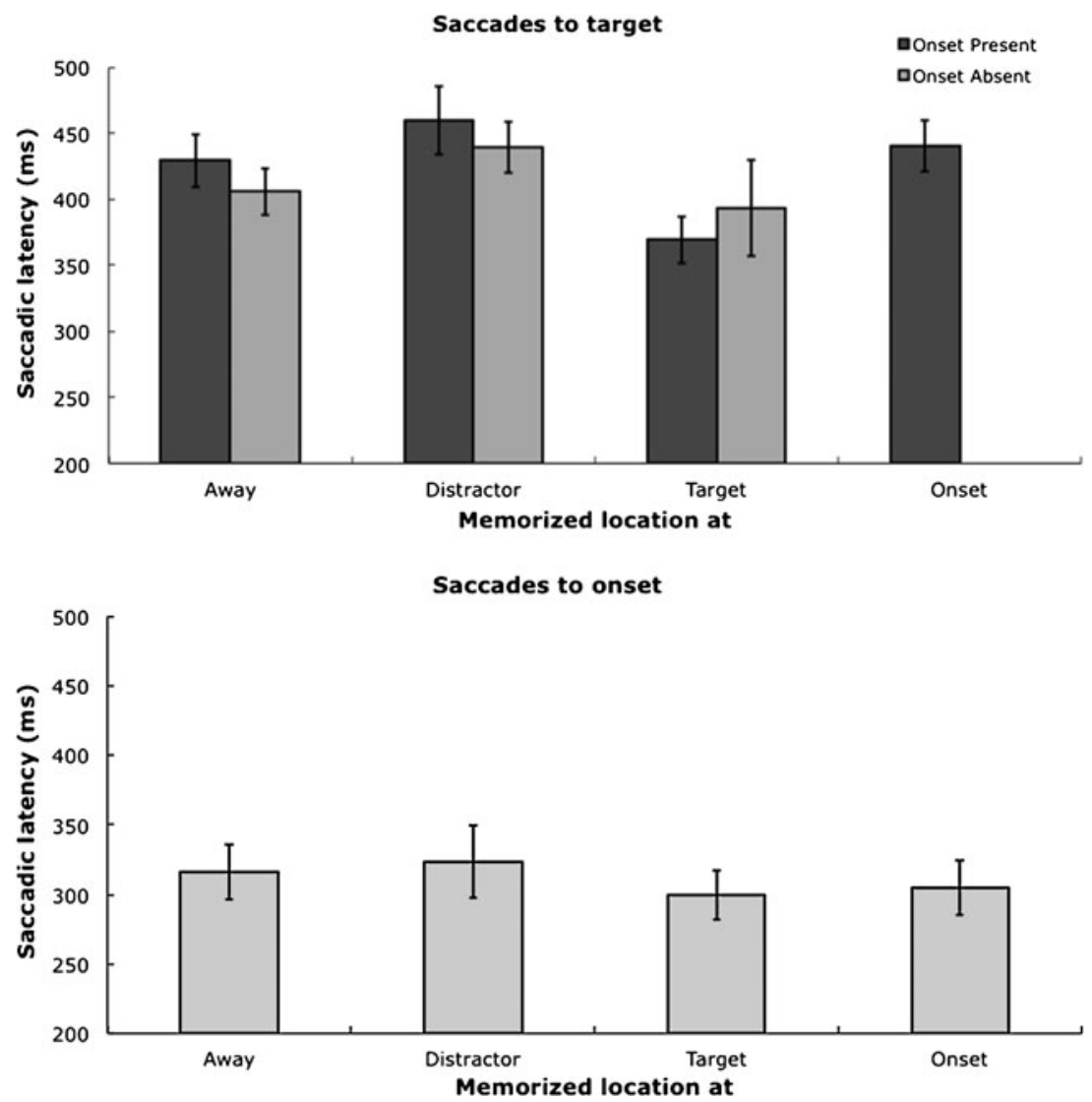

target and onset location but, by itself, spatial memory is usually not sufficient to generate a saccade.

\section{General discussion}

The three experiments presented here demonstrate that spatial working memory can influence both voluntary and involuntary saccades. This occurs when attention should be focused on the current fixation location in order to await a cue (Experiments 1 and 2) but also when attention should be spread across the display to localize a unique target item (Experiment 3).

Furthermore, these results provide evidence for attentional enhancement at locations in memory. This is presumably an endogenous effect of memory rehearsal, which is similar to the endogenous (i.e., top-down) goal of fixating the search target. Saccades to the task-irrelevant onset are exogenous because they are driven by the environment (i.e., they are generated from the bottom up). Enhancement is largest for task-relevant (targets) or salient search objects (onsets). Only in Experiment 2 did search distractors, which were not salient within the display, receive more initial fixations when colocated with the memorized location, and even that effect was small (an increase from 3.6 \% to $5.4 \%$ ). This demonstrates that attentional enhancement alone is rarely enough to attract the eyes and must be coupled with a visually salient or task-relevant object to be facilitatory. No evidence was found for a slowing of saccades to the onset, search target, or cued target when they were fixated, nor was there evidence for fewer saccades made to the abrupt onset when it coincided with the location in memory.

The effect of attentional enhancement stems from maintaining locations in spatial working memory, which is an active process that leads to attentional enhancement at the memorized location (Awh et al., 1998). This active maintenance likely enabled object processing as soon as it appeared in the display, so eye movements to the target were made more quickly. This was not found for saccades to the onset, however. This may be due to the fact that onsets have a separate, shorter pathway through the superior colliculus to saccade programming areas in the brain. From this, it is likely that spatial memory is unable to speed up the programming of an involuntary saccade; in essence, there is a ceiling effect in performance. A theoretical possibility is that spatial memory enhances processing for cortical pathways responsible for only a minority of onset-driven saccades (Sumner, Adamjee, \& Mollon, 2002) and does not enhance processing for collicular pathways.

This theory also provides an explanation for another result. When the memorized location and onset coincided, there were fewer fixations to the target; in other words, 
onsets were more likely to "steal" saccades from the target. However, the reverse was not true: When the memorized location and search target coincided, there were not fewer fixations to the onset. Since onsets are processed by the shorter collicular pathway, the saccade signal for the target occurs too late to guide the eyes, even with attentional enhancement. Support for this explanation comes from the latencies to the onset, which are substantially shorter than those to the memorized target.

Nowhere in the data is there support for the inhibition of saccades toward the memorized location; past findings of inhibition have been explained as a mechanism that protects the memory store (Belopolsky \& Theeuwes, 2009; Theeuwes et al., 2005). Inhibition would predict fewer fixations to the abrupt onset or longer saccadic latencies to onsets and targets when they were at the memorized location, and this was not found. Likewise, we found no evidence that the eyes were repelled away from a memorized location, since saccadic deviations for memorized targets or onsets were no greater than when the memorized location occurred at the target. Additionally, one would have expected decreased accuracy at remembering the memorized location when the location coincided with the target, since the eyes had to fixate directly on the target to complete the trial. Results showed the opposite, however: Memory accuracy in Experiments 2 and 3 improved when the eyes fixated on the target that coincided with the memorized location.

This increase in memory accuracy did not occur when the first fixation landed on the abrupt onset at the memorized location. Participants are often unaware that they have even made saccades to the onset (Belopolsky, Kramer, \& Theeuwes, 2008), possibly due to their involuntary nature. The lack of conscious awareness of making a saccade to the abrupt onset likely prevented a refresh of the location memory trace, which would have led to no improvement in memory accuracy. Alternatively, the covert shift of attention to the saccade might have been so brief as to not have an effect on spatial memory (Peterson et al., 2004). It is also possible that the effect of onsets on saccade programming is limited and does not spread to spatial memory processes (but see Peterson et al., 2004, for evidence showing that covert attention precedes involuntary saccades to onsets).

An important question from these results is why there was a failure to replicate the Belopolsky and Theeuwes (2009) inhibition results, especially in Experiment 1. One possibility is that our saccade latencies were roughly 100 $150 \mathrm{~ms}$ quicker, and perhaps IOR did not have time to take effect (Klein, Castel, \& Pratt, 2006; Posner \& Cohen, 1984). IOR takes time to develop, and it might be that for a period of time, IOR is able to overwhelm the excitatory effects of spatial working memory (see Eimer \& Schlaghecken, 2002, for an analogous effect for response priming). Hinting at this are recent results by Belopolsky and Theeuwes (2012) demonstrating that endogenous cuing crosses over from saccade facilitation to saccade inhibition shortly after covert attention has been shifted. Clearly, future research will seek out the locus of these inhibition and priming effects. Nonetheless, results from both labs clearly demonstrate that holding a location in working memory can affect saccade programming.

The general result of the experiments presented here is that spatial memory led to enhancement of the visual system at memorized locations, facilitating overt shifts of attention during the time span between memory encoding and memory test. This enhancement led to more saccades to objects that shared the position of the location in memory, no matter whether the saccade was a voluntary one toward the target or an involuntary saccade toward the abrupt onset. In some cases, the saccade is even made sooner, as in the case where the target coincided with the memorized location. This illustrates how spatial memory has the ability to assist the typically slow goal-driven saccade to the target in drawing the eyes.

Author Note The authors thank our lab members for helpful comments on the manuscript and, in particular, Allison Sleeman, Bobby Azarian, Eric Blumberg, and Melissa Smith for their help with Experiments 1 and 2. This work was adapted from a portion of J. H. Wong's doctoral dissertation and was funded by a Department of Defense SMART scholarship to J. H. Wong. Correspondence concerning this article should be addressed to Jason H. Wong, Naval Undersea Warfare Center, Building 1171/2, Newport, RI 02841 (email: jason.h.wong@navy.mil).

\section{References}

Awh, E., Jonides, J., \& Reuter-Lorenz, P. A. (1998). Rehearsal in spatial working memory. Journal of Experimental Psychology. Human Perception and Performance, 24(3), 780-790.

Awh, E., Jonides, J., Smith, E. E., Buxton, R. B., Frank, L. R., Love, T., Wong, E. C., \& Gmeindl, L. (1999). Rehearsal in spatial working memory: Evidence from neuroimaging. Psychological Science, 10(5), 433-437.

Belopolsky, A. V., Kramer, A. F., \& Theeuwes, J. (2008). The role of awareness in processing of oculomotor capture: Evidence from event-related potentials. Journal of Cognitive Neuroscience, 20, 2285-2297.

Belopolsky, A. V., \& Theeuwes, J. (2009). Inhibition of saccadic eye movements to locations in spatial working memory. Attention, Perception, \& Psychophysics, 71(3), 620-631.

Belopolsky, A. V., \& Theeuwes, J. (2012). Updating the premotor theory: The allocation of attention is not always accompanied by saccade preparation. Journal of Experimental Psychology. Human Perception and Performance, 38, 902-914.

Cheal, M. L., \& Lyon, D. R. (1991a). Central and peripheral precuing of forced-choice discrimination. Quarterly Journal of Experimental Psychology, 43A, 859-880.

Cheal, M., \& Lyon, D. R. (1991b). Importance of precue location in directing attention. Acta Psychologica, 76, 201-211.

Corbetta, M. (1998). Frontoparietal cortical networks for directing attention and the eye to visual locations: Identical, independent, 
or overlapping neural systems? Proceedings of the National Academy of Sciences, 95(3), 831-838.

Cowan, N. (1995). Attention and memory: An integrated framework. New York: Oxford University Press.

Curtis, C. E., \& Connolly, J. D. (2008). Saccade preparation signals in the human frontal and parietal cortices. Journal of Neurophysiology, 99, 133-145.

Deubel, H., \& Schneider, W. X. (1996). Saccade target selection and object recognition: Evidence for a common attentional mechanism. Vision Research, 36(12), 1827-1837.

Eimer, M., \& Schlaghecken, F. (2002). Links between conscious awareness and response inhibition: Evidence from masked priming. Psychonomic Bulletin and Review, 9, 514-520.

Everling, S., Dorris, M. C., \& Munoz, D. P. (1998). Reflex suppression in the anti-saccade task is dependent on prestimulus neural processes. Journal of Neurophysiology, 80, 1584-1589.

Everling, S., \& Munoz, D. P. (2000). Neuronal correlates for preparatory set associated with pro-saccades and anti-saccades in the primate frontal eye field. Journal of Neuroscience, 20, 387-400.

Hoffman, J. E., \& Subramaniam, B. (1995). The role of visualattention in saccadic eye-movements. Perception \& Psychophysics, 57(6), 787-795.

Ikkai, A., \& Curtis, C. E. (2008). Cortical activity time locked to the shift and maintenance of spatial attention. Cerebral Cortex, 18, 1384-1394.

Ikkai, A., \& Curtis, C. E. (2010). Common neural mechanisms supporting spatial working memory, attention, and motor intention. Neuropsychologia, 49, 1428-1434.

Klein, R. M. (2000). Inhibition of return. Trends in Cognitive Sciences, 4(4), 138-147.

Klein, R. M., Castel, A. D., \& Pratt, J. (2006). The effects of memory load on the time course of inhibition of return. Psychonomic Bulletin \& Review, 13, 294-299.

Müller, H. J., \& Rabbitt, P. M. A. (1989). Reflexive and voluntary orienting of visual attention: Time course of activation and resistance to interruption. Journal of Experimental Psychology. Human Perception and Performance, 15, 315-330.
Olivers, C. N. L., Meijer, F., \& Theeuwes, J. (2006). Feature-based memory-driven attentional capture: Visual working memory content affects visual attention. Journal of Experimental Psychology. Human Perception and Performance, 32(5), 1243-1265.

Peterson, M., Kramer, A., \& Irwin, D. E. (2004). Covert shifts of attention precede involuntary eye movements. Perception \& Psychophysics, 66(3), 398-405.

Posner, M. I., \& Cohen, Y. (1984). Components of visual orienting. In H. Bouma \& D. Bonwhuis (Eds.), Attention and performance X: Control of language processes (pp. 551-556). Hillsdale, N. J: Erlbaum.

Postle, B. R., Awh, E., Jonides, J., Smith, E. E., \& D'Esposito, M. (2004). The where and how of attention-based rehearsal in spatial working memory. Cognitive Brain Research, 20(2), 194-205.

Rizzolatti, G., Riggio, L., Dascola, I., \& Umiltà, C. (1987). Reorienting attention across the horizontal and vertical meridians: Evidence in favor of a premotor theory of attention. Neuropsychologia, 25, $31-40$.

Srimal, R., \& Curtis, C. E. (2007). Persistent neural activity during the maintenance of spatial position in working memory. NeuroImage, 39, 455-468.

Sumner, P., Adamjee, T., \& Mollon, J. D. (2002). Signals invisible to the collicular and magnocellular pathways can capture visual attention. Current Biology, 12(15), 1312-1316.

Theeuwes, J., Olivers, C. N. L., \& Chizk, C. L. (2005). Remembering a location makes the eyes curve away. Psychological Science, 16 (3), 196-199.

Treue, S. (2001). Neural correlates of attention in primary visual cortex. Trends in Neuroscience, 24, 295-300.

Wong, J. H., \& Peterson, M. S. (2011). The interaction between memorized objects and abrupt onsets in oculomotor capture. Attention, Perception, \& Psychophysics, 73(6), 1768-1779.

Yantis, S., \& Jonides, J. (1990). Abrupt visual onsets and selective attention: Voluntary versus automatic allocation. Journal of Experimental Psychology. Human Perception and Performance, 16, $121-134$. 